\title{
Eficacia del Programa de Habilidades para la Vida en Adolescentes Escolares de Huancavelica, Perú
}

\section{Determining the efficacy of a high-school life-skills' programme in Huancavelica, Peru}

\author{
Raúl Choque-Larrauri ${ }^{1}$ y Jesús Lorenzo Chirinos-Cáceres ${ }^{2}$
}

1 Universidad Nacional Mayor de San Marcos, Lima, Perú. raul.choque@pucp.edu.pe

2 Facultad de Salud Pública y Administración, Universidad Peruana Cayetano Heredia, Lima, Perú.

Recibido 29 Septiembre 2008/Enviado para Modificación 8 Abril 2009/Aceptado 20 Abril 2009

\section{RESUMEN}

Objetivo Determinar la eficacia de un programa educativo de habilidades para la vida en el marco de las escuelas promotoras de la salud, en adolescentes escolares de una institución educativa del distrito de Huancavelica, Perú, implementado en el año escolar 2006.

Métodos Investigación experimental, con preprueba y posprueba, con grupo control no equivalente. Fueron estudiados 284 estudiantes adolescentes de educación secundaria. Las variables analizadas fueron comunicación, autoestima, asertividad, toma de decisiones, sexo y edad.

Resultados Hubo un incremento significativo en el desarrollo de las habilidades de comunicación y asertividad en los estudiantes del grupo experimental. No se encontraron diferencias significativas en el desarrollo de la habilidad de toma de decisiones y la autoestima.

Discusión El programa educativo de habilidades para la vida es efectivo en un año escolar en el aprendizaje y desarrollo de las habilidades de comunicación y asertividad, sin embargo la autoestima y la habilidad de toma de decisiones no mostraron un cambio estadísticamente significativo por lo que es necesario reorientar su implementación. Asimismo el programa educativo de habilidades para la vida se debe desarrollar durante toda la educación secundaria a fin de notar cambios significativos en las habilidades estudiadas.

Palabras Clave: Aspectos psicosociales, educación, entrenamiento (fuente: DeCS, BIREME).

\section{ABSTRACT}

Objective Determining the efficacy of a life-skills' programme within the context of a school health promotion programme using teenagers from a high-school in the district of Huancavelica, Peru during school year 2006.

Methods This was non-equivalent experimental research with pre-test and post-test. The subjects consisted of 284 high school students. The variables analyzed were 
communication, self esteem, assertiveness, decision making, sex and age. Results There was a significant increase in the experimental group's communication and assertiveness skills' development. There were no significant differences in decision-making and self-esteem skills.

Discussion The life-skills' programme was effective during one school year, especially in terms of learning and developing communication and assertiveness skills. However, self-esteem and decision-making skills did not present a statistically significance difference. Programme implementation must thus be redirected and the life-skills' programme should be implemented throughout all high-school years.

Key Words: Psychosocial aspects, education, training (source: MeSH, NLM).

$\mathrm{E}$ s extensa la literatura que trata de conceptualizar y definir qué entiende la comunidad científica por el término de habilidades para la vida, encontrándose que aún no existe una aceptación universalmente consensuada o definición. Lo que si es un consenso es su ámbito de intervención que es el aspecto socio afectivo de las personas $(1,2)$.

Otro aspecto importante a tomar en cuenta es la existencia de varios términos referidos al campo de las habilidades para la vida las cuales se presentan en la bibliografía científica. Estos términos son el de habilidades sociales, habilidades psicosociales y competencia social. Cabe señalar que en muchos casos aún sólo se utiliza el término de habilidades sociales.

Para conceptualizar el término de habilidades para la vida en esta investigación, fue necesario en primer lugar tomar en cuenta la definición del término habilidad que es una capacidad y disposición para hacer algo y una de las cosas que una persona ejecuta con gracia y destreza (3). Asimismo el término habilidad sugiere la capacidad de llevar a la práctica satisfactoriamente una acción en una situación determinada, de ahí que las habilidades sean infinitas en cuanto a su variedad e interpretación en situaciones de aplicación y algunas habilidades requieren de mayor o menor grado de elaboración cognitiva según sea el caso (4).

El término de habilidades para la vida ha tenido una evolución importante, desde términos como asertividad, habilidades sociales, competencia psicosocial, competencia social entre otras. Uno de los términos más difundidos es el de habilidades sociales del cual se atribuye su origen a Salter, uno de los padres de la terapia de la conducta quien en su libro Conditioned Reflex Therapy (1949) describió seis técnicas para aumentar la expresivi- 
dad de las personas las cuales eran las siguientes: la expresión verbal y facial de las emociones, el empleo deliberado de la primera persona al hablar, el estar de acuerdo cuando se reciben alabanzas, el expresar desacuerdo y la improvisación y actuación espontáneas (5).

En relación al campo de intervención de las habilidades para la vida, los comienzos de la investigación provienen del campo de la terapia de la conducta y es a partir de los años 90 que recién se dirigió al campo educativo. Por lo cual a partir de ese año se le dio importancia al desarrollo de las habilidades para la vida en los colegios por ser espacios ideales de aprendizaje (6). Es de esta manera que desde hace más de 16 años hay una importante intervención en el desarrollo de las habilidades para la vida en la educación formal, habiéndose logrado incluso su incorporación en la currícula educativa de la educación básica como es el caso de Colombia (7).

Con la finalidad de conceptualizar el término de habilidades para la vida en esta investigación, efectuamos previamente una sistematización de las definiciones y conceptualizaciones sobre habilidades para la vida, correspondientes a científicos, investigadores e instituciones especializadas en el tema, que a lo largo de los últimos años han desarrollo y que las recogemos para fines de análisis:

Las habilidades para la vida son destrezas para conducirse de cierta manera, de acuerdo con la motivación individual y el campo de acción que tenga la persona, dentro de sus posibilidades sociales y culturales. Son un eslabón o "puente" entre los factores motivadores del conocimiento, las actitudes y los valores, y el comportamiento o estilo de vida saludable (7).

Las habilidades para la vida promueven el desarrollo de factores de protección comunes a diversos problemas psicosociales y que facilitan a los estudiantes la tarea de enfrentar con éxito las exigencias, demandas y desafíos de la vida diaria; reconoce el rol de las competencias psicosociales tales como: la autoestima, la asertividad, las habilidades para la toma de decisiones, el pensamiento creativo crítico y reflexivo, el manejo de emociones y sentimientos, la comunicación efectiva y la autonomía moral (7).

Las habilidades para la vida son el conjunto de habilidades que permiten a las personas actuar de manera competente y habilidosa en las distintas situa- 
ciones de la vida cotidiana y con su entorno, favoreciendo comportamientos saludables en las esferas física, psicológica y social. Comprende tres categorías de habilidades: sociales, cognitivas y para el manejo de las emociones (8).

Las habilidades para la vida son habilidades personales, interpersonales, cognitivas y físicas que permiten a las personas controlar y dirigir sus vidas, desarrollando la capacidad para vivir con su entorno y lograr que éste cambie. Como ejemplos de habilidades para la vida individuales se pueden citar la toma de decisiones y solución de problemas, el pensamiento creativo y crítico, el conocimiento de sí mismo y la empatía, las habilidades de comunicación y de relación interpersonal y la capacidad para hacer frente a las emociones y manejar el estrés (9).

Las habilidades para la vida son un enfoque que desarrolla destrezas para permitir que los adolescentes adquieran las aptitudes necesarias para el desarrollo humano y para enfrentar en forma efectiva los retos de la vida diaria. Identifican tres clases de habilidades: 1) habilidades sociales o interpersonales (incluyendo comunicación, habilidades de rechazo, asertividad, agresividad y empatía), 2) habilidades cognitivas (incluyendo la toma de decisiones, el pensamiento crítico y la auto evaluación), y 3) habilidades para manejar emociones, incluyendo el estrés y aumento interno de un centro de control (8).

Teniendo en consideración estas definiciones tanto de instituciones y diversos científicos y especialistas sobre el tema de habilidades para la vida, la conclusión es que no hacen sino constatar la falta de consenso y de clarificación que dificulta a los profesionales del ámbito social definir qué entendemos por habilidades para la vida, por lo cual nos pareció que podía resultar enriquecedor y clarificador, como si indica en el Cuadro 1 la reconstrucción de los aspectos más significativos de la conceptualización de habilidades para la vida.

Del resultado de este análisis elaboramos el concepto de habilidades para la vida que utilizamos en esta investigación:

"Las habilidades para la vida son las capacidades y destrezas en el ámbito socioafectivo de las personas, entre ellas habilidades sociales, cognitivas y de manejo de emociones, que les permiten enfrentar con éxito las exigen- 
cias, demandas y desafíos de la vida diaria, actuando competentemente y contribuyendo al desarrollo humano".

Es importante tener en cuenta que existen una serie de clasificaciones de las habilidades para la vida. Una de estas clasificaciones establece tres grandes categorías que son las habilidades sociales o interpersonales, las habilidades cognitivas y las habilidades para el manejo de las emociones (7). Una característica importante de esta clasificación es que estas tres categorías se relacionan entre ellas e interactúan.

Cuadro 1. Componentes de las habilidades para la vida

\begin{tabular}{|c|c|c|}
\hline Contenido & Características & Consecuencias \\
\hline $\begin{array}{l}\text { - Autoestima } \\
\text { - Empatía } \\
\text { - Toma de decisi ones } \\
\text { - Comunicación efectiva } \\
\text { - Manejo de emociones y } \\
\text { sentimientos } \\
\text { - Asertividad } \\
\text { - Pensamiento creativo, } \\
\text { crítico y reflexivo } \\
\text { - Autonomía moral } \\
\text { - Relaciones interpersonales } \\
\text { - Manejo de tensiones y } \\
\text { estrés } \\
\text { - Solución de problemas y } \\
\text { conflictos }\end{array}$ & $\begin{array}{l}\text { - Son comportamientos } \\
\text { adquiridos y aprendidos } \\
\text { - Centrado en el ámbito } \\
\text { sociafectivo de la persona } \\
\text { - Desarrolla factores } \\
\text { protectores } \\
\text { - Es una educación } \\
\text { sentimental } \\
\text { - Desarrolla habilidades } \\
\text { sociales, cognitivas y para } \\
\text { manejar las emociones } \\
\text { - Favorece comportamientos } \\
\text { saludables en las esferas } \\
\text { física, psicológica y social }\end{array}$ & $\begin{array}{l}\text { - Enfrentar con éxito las } \\
\text { exigencias, demandas y } \\
\text { desafíos de la vida diaria } \\
\text { - Actuar de manera } \\
\text { competente } \\
\text { - Adquirir aptitudes } \\
\text { necesarias para el desarrollo } \\
\text { humano } \\
\text { - Comportamientos y estilos } \\
\text { de vida saludables } \\
\text { - Conseguir una mejor salud } \\
\text { mental y una existencia más } \\
\text { feliz } \\
\text { - Resolver problemas } \\
\text { inmediatos de la situación } \\
\text { - Se da dentro de sus } \\
\text { posibilidades sociales y } \\
\text { culturales }\end{array}$ \\
\hline
\end{tabular}

Las habilidades sociales son las destrezas sociales específicas requeridas para ejecutar competentemente una tarea de índole interpersonal (10). Implica un conjunto de conductas emitidas por un individuo en un contexto interpersonal que expresa los sentimientos, actitudes, deseos, opiniones o derechos de ese individuo, de un modo adecuado a la situación, respetando esas conductas de los demás, y que generalmente resuelve los problemas 
inmediatos de la situación mientras minimiza la probabilidad de futuros problemas. Las habilidades cognitivas son un conjunto de operaciones mentales, cuyo objetivo es que el estudiante integre la información adquirida a través de los sentidos en una estructura de conocimiento que tenga sentido para él (11). Las habilidades para el manejo de las emociones son los estados anímicos que manifiestan una gran actividad orgánica y que se reflejan en los comportamientos externos e internos (12).

Las habilidades para la vida en Huancavelica, Perú

En los últimos años se observa que la educación peruana ha avanzado considerablemente en la cobertura de la educación básica, tal es así que la cobertura en la educación secundaria fue del $82 \%$ (13). Sin embargo en el aspecto de la calidad educativa hay mucha postergación y esto se demuestra en los resultados de la Evaluación Nacional de Rendimiento Estudiantil llevada a cabo en el año 2004 por el Ministerio de Educación. Los resultados de esta evaluación señalaron que en educación secundaria sólo el 9,8\% de estudiantes alcanzaron el nivel de desempeño suficiente en comunicación integral y el 2,9\% de estudiantes alcanzaron el nivel de desempeño suficiente en lógico matemático (14).

Enfocándonos sobre el campo de investigación, es decir sobre las habilidades para la vida, hoy se sabe a plenitud el valor de las competencias afectivas, pues mientras millones de niños y jóvenes, desde las siete de la mañana hasta bien entrada la tarde, estudian el cuadrivio occidental: matemáticas, ciencias naturales, idiomas y ciencias sociales; nada en absoluto estudian de habilidades para la vida, fuente básica de felicidad, de disminución de la angustia y privilegio de salud (15). Además, siendo la educación integral esta no se realiza integralmente porque no se desarrolla el aprendizaje de aspectos socio afectivos y de habilidades para la vida en el ámbito de la educación, puesto que el sistema educativo está focalizado prioritariamente hacia el desarrollo de competencias en matemáticas y lenguaje, tal como se puede apreciar en la currícula educativa (16).

En este contexto, el descuido de la educación en el campo socio afectivo de los escolares es bastante alarmante y esto se demuestra en un estudio nacional realizando por la Oficina de Tutoría y Prevención Integral del Ministerio de Educación donde se presentó que en el Perú el 31,3\% de escolares presentan serias deficiencias en sus habilidades sociales (17). Es decir, de cada 100 escolares adolescentes en el Perú, 31 escolares presentan defi- 
ciencias significativas en sus habilidades sociales, entre ellas las habilidades de comunicación, habilidades para reducir la ansiedad, habilidades para la autoafirmación personal, habilidades para la afirmación de vínculos amicales y habilidades para la afirmación de vínculos sociales en general. En el caso de la Región Huancavelica los resultados de la investigación mostraron que el $58,8 \%$ de escolares presentaron serias deficiencias en sus habilidades sociales, es decir de cada 10 escolares huancavelicanos, 6 de ellos tienen deficiencias en sus habilidades sociales.

El Ministerio de Salud del Perú, viene implementado desde el año 2002 la Promoción de la Salud y en ese marco la estrategia de Escuelas Saludables que tiene como finalidad contribuir al desarrollo humano integral de los estudiantes y de la comunidad educativa mediante el fortalecimiento de acciones de promoción de la salud en las instituciones educativas (18).

Las escuelas saludables son aquellas que contribuyen a fomentar el desarrollo humano integral y las relaciones humanas constructivas y armónicas, a fin de lograr comportamientos, capacidades y competencias saludables, con un entorno físico, psicológico y social positivo para el aprendizaje. Como se indica en el Gráfico 1 el desarrollo de las habilidades para la vida comprende un proceso.

Gráfico 1. Modelo del Programa Educativo de Habilidades para la Vida

\section{ESTRUCTURA \\ PROCESO \\ RESULTADO \\ IMPACTO}

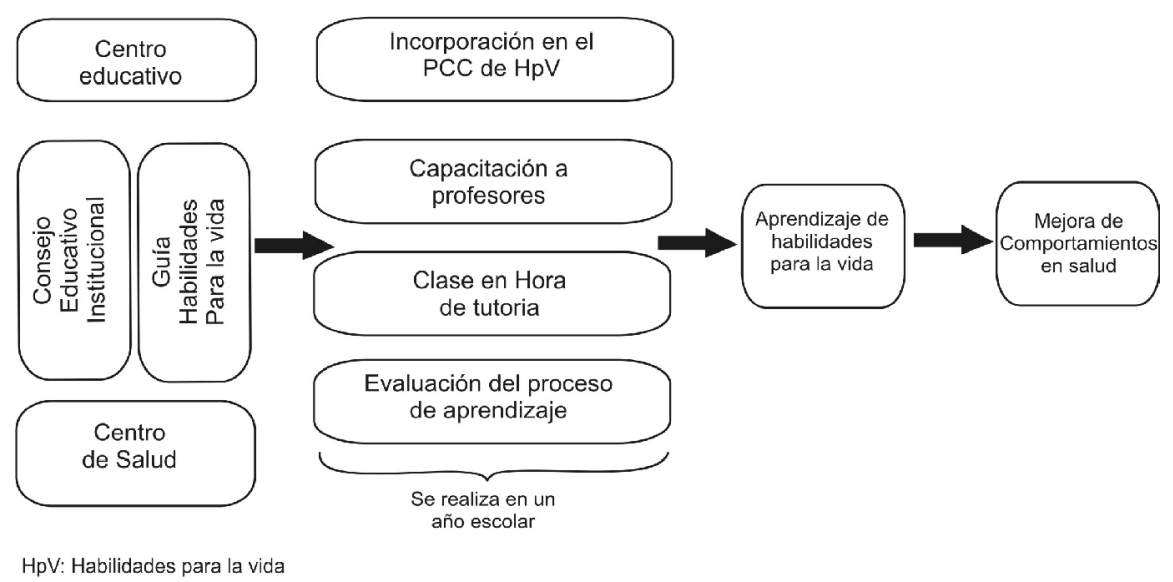

HpV: Habilidades para la vida 


\section{METODOLOGÍA}

El estudio se realizó en la Región de Huancavelica, que es la más pobre del Perú. En esta Región el 83,7 \% de la población está en pobreza y el 61,1 \% en situación de pobreza extrema. El Índice de Desarrollo Humano es de 0,4 que la cataloga en el nivel bajo de desarrollo humano. El $24 \%$ de la población es analfabeta.

La investigación fue experimental con preprueba y posprueba, con grupo de control no equivalente. La población estuvo constituida por adolescentes escolares entre 13 a 16 años de edad, varones y mujeres de $3^{\circ}, 4^{\circ}$ y $5^{\circ}$ grado de educación secundaria de dos instituciones educativas de educación secundaria estatales del distrito de Huancavelica. Se calculó la muestra con un intervalo de confianza al $95 \%$, resultando 142 para el grupo experimental y 142 para el grupo control. El Programa Educativo de Habilidades para la Vida se desarrolló en el grupo experimental. El instrumento utilizado fue la lista para evaluación de habilidades para la vida (19) (20).

\section{RESULTADOS}

Como se indica en la Tabla 1 en las características socio-demográficas no hubo diferencias significativas entre los estudiantes del grupo experimental y el grupo de control. Observándose similares distribuciones en cuanto a sexo, edad, personas con la que viven, situación de repitencia, escolaridad de las madres y situación de trabajo entre ambos grupos, por lo cual la comparación fue factible.

Resultados de habilidades para la vida

Se observó que en la medición basal de las habilidades para la vida (asertividad, comunicación, autoestima y toma de decisiones) al comparar el grupo experimental en relación al grupo de comparación no se encontraron diferencias. Mientras que en la medición final se observaron diferencias en ambos grupos, a favor del grupo experimental en las habilidades de asertividad y comunicación más no en las habilidades de autoestima y toma de decisiones tal como se indica en la Tabla 2. 
Tabla 1. Características socio-demográficas de ambos grupos (Pre-Test)

\begin{tabular}{|c|c|c|c|c|c|c|}
\hline \multirow[t]{2}{*}{ Variables } & \multicolumn{2}{|c|}{$\begin{array}{c}\text { Grupo } \\
\text { experimental } \\
N=142\end{array}$} & \multicolumn{2}{|c|}{$\begin{array}{c}\text { Grupo de } \\
\text { comparación } \\
N=142\end{array}$} & \multirow[t]{2}{*}{$x^{2}(g l)$} & \multirow[t]{2}{*}{ Valor $p$} \\
\hline & $\%$ & $\mathrm{~N}$ & $\%$ & $\mathrm{~N}$ & & \\
\hline Sexo & & & & & $x^{2}(1)=0,100$ & 0,276 \\
\hline Masculino & 55,6 & 79 & 51,4 & 73 & & \\
\hline Femenino & 44,4 & 63 & 48,6 & 69 & & \\
\hline Lengua materna & & & & & $x^{2}(1)=0,140$ & 0,402 \\
\hline Castellano & 35,9 & 51 & 33,8 & 48 & & \\
\hline Quechua & 64,1 & 91 & 66,2 & 94 & & \\
\hline Personas con las que viven & & & & & $x^{2}(3)=2,498$ & 0,476 \\
\hline Ambos padres & 67,6 & 96 & 74,6 & 106 & & \\
\hline Uno de los padres & 23,9 & 34 & 19,0 & 27 & & \\
\hline Otros familiares & 7,0 & 10 & 4,2 & 6 & & \\
\hline Solo & 1,4 & 2 & 2,1 & 3 & & \\
\hline Situación de repitencia & & & & & $x^{2}(1)=0,044$ & 1,000 \\
\hline Si repite & 9,2 & 13 & 8,5 & 12 & & \\
\hline No repite & 90,8 & 129 & 91,5 & 130 & & \\
\hline Escolaridad de las madres & & & & & $x^{2}(3)=0,384$ & 0,944 \\
\hline Analfabeta & 24,6 & 35 & 22,5 & 32 & & \\
\hline Primaria & 57,0 & 81 & 60,6 & 86 & & \\
\hline Secundaria & 14,8 & 21 & 13,4 & 19 & & \\
\hline Superior & 3,5 & 5 & 3,5 & 5 & & \\
\hline Situación de trabajo & & & & & $x^{2}(1)=3,662$ & 0,060 \\
\hline Si trabaja & 4,2 & 6 & 0,7 & 1 & & \\
\hline \multirow[t]{2}{*}{ No trabaja } & 95,8 & 136 & 99,3 & 141 & & \\
\hline & Media & DS & Media & DS & $\mathrm{t}(\mathrm{gl})$ & Valor $p$ \\
\hline Edad & 14,66 & 2,0 & 14,9 & 1,7 & $t(282)=1,606$ & 0,141 \\
\hline
\end{tabular}

Tabla 2. Resultados de habilidades para la vida en el momento final

\begin{tabular}{lrrrrrr}
\hline $\begin{array}{c}\text { Habilidades para la } \\
\text { vida }\end{array}$ & $\begin{array}{c}\text { Grupo } \\
\text { experimental } \\
N=142\end{array}$ & \multicolumn{2}{c}{$\begin{array}{c}\text { Grupo de } \\
\text { comparación } \\
N=142\end{array}$} & $t$ & $\begin{array}{c}\text { Valor } \\
p\end{array}$ \\
\hline Media & DS & Media & DS \\
Asertividad & 3,3 & 1,1 & 3,0 & 1,2 & 2,3 & $0.01^{*}$ \\
Comunicación & 3,5 & 1,2 & 3,2 & 1,2 & 1,9 & $0.04^{*}$ \\
Autoestima & 3,1 & 1,0 & 2,9 & 1,1 & 1,9 & 0.05 \\
\hline Toma de decisiones & 2,8 & 1,1 & 2,6 & 1,0 & 1,1 & 0.26 \\
\hline
\end{tabular}

* Diferencia estadísticamente significativas a favor del grupo experimental.

Los resultados de las habilidades de autoestima y toma de decisiones no mostraron diferencias estadísticamente significativas. De estos resultados se establece que el aprendizaje de las habilidades de autoestima y toma de decisiones no fue eficaz. 
Variables sexo y edad

En el aprendizaje de las habilidades para la vida según la variable sexo, no encontramos una diferencia estadísticamente significativa en el grupo experimental, al comparar los resultados de la línea basal y de la medición final. Asimismo, en el aprendizaje de las habilidades para la vida según la variable edad, no encontramos una diferencia estadísticamente significativa en el grupo experimental, al comparar los resultados de la línea basal y de la medición final.

\section{DISCUSIÓN}

En relación con las habilidades para la vida

Los resultados de la investigación muestran que hay diferencias estadísticamente significativas en el aprendizaje de las habilidades de asertividad y comunicación. Una razón que tomamos en consideración sobre estos resultados es que estas habilidades tienen una mayor facilidad en su aprendizaje, puesto que se aplican en todo momento y no requieren de procesos previos y complejos. Es importante mencionar además que la metodología educativa aplicada es la participativa la que conlleva una interacción alta entre los estudiantes y el profesor lo que obviamente favorece un mejor nivel de comunicación entre los estudiantes.

Al respecto en la evaluación del programa escolar de habilidades para vivir en Costa Rica, se encontró en el período de un año escolar incrementos en los dominios de relaciones interpersonales, es decir en las habilidades de comunicación y empatía (21). El estudio también demostró que no hubo diferencias estadísticamente significativas en el aprendizaje de las habilidades de autoestima y toma de decisiones.

Por otra parte la evaluación del programa de habilidades para la vida "La Aventura de la Vida", desarrollada por EDEX de España, demostró que la autoestima y la habilidad de decisión reflexiva tuvo variación significativa, luego de haberse realizado durante dos y tres años respectivamente y que al año de intervención no hubo variación significativa.

De estos estudios podemos establecer que la autoestima y la habilidad de toma de decisiones son más complejas y que requieren de una serie de procesos y etapas previas, así como una mayor temporalidad para su desarrollo. 
En relación a la habilidad de toma de decisiones se menciona que esta habilidad es un proceso complicado que un simple proceso racional, puesto que requiere de un análisis afectivo, emocional y cognitivo (22). Asimismo para la toma de decisiones, especialmente bajo condiciones de estrés, involucran habilidades de pensamiento cognitivo (identificación de asuntos o problemas, determinación de metas, generación de soluciones alternativas, imaginación de posibles consecuencias) y habilidades para enfrentar emociones (calmarse a sí mismo en situaciones de estrés, escuchar con exactitud y determinar la mejor opción) (23).

Para desarrollar la habilidad de toma de decisiones se requiere pensamiento crítico para evaluar y detectar un problema o una situación que requiere tomar una decisión. También se requiere pensamiento creativo para analizarlo y sintetizarlo, además de la capacidad de resolver un problema para encontrar alternativas de solución. La toma de decisiones implica además elegir la mejor opción para solucionar una dificultad y tener una facultad para comprender entre varias opciones cuál es la mejor.

En relación a la autoestima se requiere de un proceso más amplio de desarrollo puesto que éste componente de la personalidad está constituida de varias habilidades como son: autoconocimiento, autoconcepto, autoevaluación y autorrespeto (22). Cada una de ellas requiere trabajarse en el aula y en una temporalidad suficiente que permitan consolidarse.

De las evaluaciones realizadas se establece que la autoestima y la habilidad de toma de decisiones requieren de un trabajo con una mayor temporalidad, así como de procesos cognitivos, emocionales y afectivos más complejos.

En relación con las variables sexo y edad

El resultado de este estudio establece que la variable de sexo no mostró una variación estadísticamente significativa. Otros estudios no tomaron en cuenta esta variable por lo cual no se puede establecer antecedentes

Agradecimientos. Agradecemos a los profesores y estudiantes de las instituciones educativas Ramón Castilla Marquesado y César Vallejo del distrito de Huancavelica, Perú por habernos brindado el apoyo para aplicar esta investigación. A la Dra. Inés Verónica Bustamante Chávez, de la Universidad Peruana Cayetano Heredia, por su apoyo científico. 


\section{REFERENCIAS}

1. Bravo A, Martínez V, Mantilla L. Habilidades para la Vida, una propuesta educativa para convivir mejor. Bogotá: Fe y Alegría; 2003.

2. Mangrulkar L, Vince C, Posner M. Enfoque de habilidades para la vida para un desarrollo saludable de niños y adolescentes. Organización Panamericana de la Salud [Internet]. Disponible en: http://www.paho.org/Spanish/HPP/HPF/ADOL/Habilidades.pdf. Consultado en Mayo del 2006.

3. Real Academia Española [Internet]. Disponible en: http://www.rae.es . Consultado en Octubre del 2006.

4. Glazman R. Las caras de la evaluación educativa. México: Universidad Autónoma de México; 2005.

5. Paula I. Habilidades sociales: educar hacia la autorregulación. Barcelona: Instituto de Ciencias de la Educación de la Universidad de Barcelona; 2000.

6. Birrell R, Orley J, Evans V, Lee J, Sprunger B, Pellaux D. Life Skills Education for Children and Adolescents in Schools. Géneva Switzerland: World Health Organization; 1997.

7. Ministerio de Educación. Tutoría y orientación educativa en la educación secundaria. Lima: Ministerio de Educación; 2005.

8. Ministerio de Salud. Orientaciones técnicas del tema de habilidades para la vida. Lima: Dirección General de Promoción de la Salud; 2005.

9. Organización Mundial de la Salud. Glosario de Promoción de la Salud [Internet]. Disponible en: http://www.bvs.org.ar/pdf/glosario_sp.pdf . Consultado en Junio del 2006.

10. Caballo V. Manual de evaluación y entrenamiento de las habilidades sociales. 3ra. Edición. Madrid: Siglo XXI; 1999.

11. Arredondo V, Pérez G, Aguirre M. Didáctica general. México: Limusa Editores; 2004.

12. Marti E. Psicología del desarrollo: el mundo del adolescente. México: Alfaomega Grupo Editor; 2005.

13. Ministerio de Educación. Evaluación de las políticas en educación durante el quinquenio 2001-2005. Lima: Oficina de Planificación Estratégica y Medición de la calidad educativa; 2006.

14. Ministerio de Educación. Evaluación Nacional de rendimiento estudiantil 2004. Lima: Oficina de Estadística Educativa; 2006.

15. De Zubiría M. Enfoques pedagógicos y didácticas contemporáneas. Bogotá: Fundación Internacional de Pedagogía Conceptual; 2003.

16. Valles A. Habilidades sociales e inteligencia emocional para el afrontamiento de la conflictividad [Internet]. Disponible en: http://www.bibliotecasocial.net/default.asp . Consultado en Octubre del 2006.

17. Ministerio de Educación. Habilidades sociales en escolares del Perú. Lima: Oficina de Tutoría y Prevención Integral; 2003.

18. Ministerio de Salud. Programa de Promoción de la Salud en las Instituciones Educativas. Lima: Dirección General de Promoción de la Salud; 2005.

19. Arévalo M, Cortez E, Escalante M, Uribe R, Velásquez W. Manual de habilidades sociales en adolescentes escolares. Lima: Instituto Nacional de Salud Mental Honorio Delgado Hideyo Noguchi; 2005.

20. Cárdenas J. Guía para el desarrollo de la capacidad de toma de decisiones. Lima: Unidad de Desarrollo Curricular y Recursos Educativos de Educación Secundaria del Ministerio de Educación; 2006. 
21. Bejarano J, Ugalde F, Morales D. Evaluación de un programa escolar en Costa Rica basado en habilidades para vivir [Internet]. Disponible en: http:// socidrogalcohol.psiquiatria.com/adicciones/vol_17/revista1.html\#edu . Consultado en Junio del 2006.

22. Beyth-Marom R, Fischhoff B, Jacobs M, Furby L. Teaching Decision-Making to Adolescents: A Critical Review. New York: Carnegie Corporation of New York; 1989.

23. Elías M, Kress J. Social Decisión-Making and Life Skills Development: A Critical Thinking Approach to Health Promotion in Middle School. Journal of School Health 1994; 64(2): 62-66.

24. Mateo J. La evaluación educativa, su práctica y otras metáforas. México: Alfaomega Grupo Editor; 2000. 